\title{
Математическое моделирование хозяйства Центрально-Черноземного региона
}

\author{
Ю.М. Фетисов, А.Э. Крупко \\ Воронежский государственный университет, Российская Федераџия \\ (394018, г. Воронеж, Университетская пл., 1)
}

\begin{abstract}
Аннотация: Цель - выявить особенности моделирования хозяйства Центрально-Черноземного региона в условиях неравномерности и неопределенности социально-экономического развития.

Maтериалы и методы. В статье были применены структурно-системный, математический и аналитический методы исследования. При моделировании хозяйства ЦЧР мы использовали различные виды матричных предикторных моделей. В статье использована статистическая информация, представленная в официальных изданиях Федеральной службы государственной статистики - «Регионы России» за 2019 год. Для выполнения расчетов статистические данные были обобщены, систематизированы и структурированы.

Результаты и обсуждение. Были рассчитаны показатели развития хозяйств региона, построены модели разного типа с помощью косвенных приростов по коротким рядам показателей развития хозяйства на основе детерминированного матричного предиктора, отмечены их недостатки и преимущества, проведена оценка точности моделей.

Заключение. Математическое моделирование хозяйства региона в условиях неравномерности и неопределенности социально-экономического развития на основе коротких и сверхкоротких рядов - одно из основных направлений моделирования сложных многомерных социально-экономических региональных процессов как отдельный метод и как часть комплексного математического моделирования социально-экономического развития региона.
\end{abstract}

Ключевые слова: Центрально-Черноземный район, развитие, неравномерность, неопределенность, детерминированный матричный предиктор, абсолютный прирост, косвенный темпы прироста.

Для цитирования: Фетисов Ю.М., Крупко А.Э. Математическое моделирование хозяйства Центрально-Черноземного региона // Вестник Воронежского государственного университета. Серия: География. Геоэкология, 2021, № 4, с. 50-59. DOI: https://doi.org/10.17308/geo.2021.4/3750

\section{ВВЕДЕНИЕ}

Для Центрально-Черноземного экономического района (ЦЧР) характерна значительная неравномерность социально-экономического развития под воздействием внутренних и внешних факторов. Если в советское время больше действовали внутренние факторы, которые в заметной степени под воздействием жесткого управления обеспечивали стабильность развития, то в настоящее время ситуация иная. Так как хозяйство района является открытой системой, то на внутренний дисбаланс накладываются факторы внешнего воздействия (санкции, цены на нефть и т.д.). Внутренний дисбаланс территориально-отраслевой структуры ЦЧР (недостаточное развитие многих отраслей и производств) снижает возможности устойчивого функционирования хозяйства и обусловливает ориентацию предприятий района на продукцию других регионов и импорт. При этом для областей ЦЧР и района в целом характерна значительная неравномерность социально-экономического развития (рис. 1).

Средняя динамика роста внутреннего регионального продукта (ВРП) за 2010-2017 годы составила в Белгородской области 106,3\%, в Воронежской $104,9 \%$, в Курской - 104,9\%, в Липецкой - 103,1\%, в Тамбовской области - $105,2 \%$. Как видим на рисунке 1, средний уровень динамики ВРП только в единичных случаях близок к реальной динамике. В Липец-

(C) Фетисов Ю.М., Крупко А.Э., 2021

Крупко Анатолий Эммануилович, e-mail: glomer-a@mail.ru

(c) (i) Контент доступен под лицензией Creative Commons Attribution 4.0 License. 


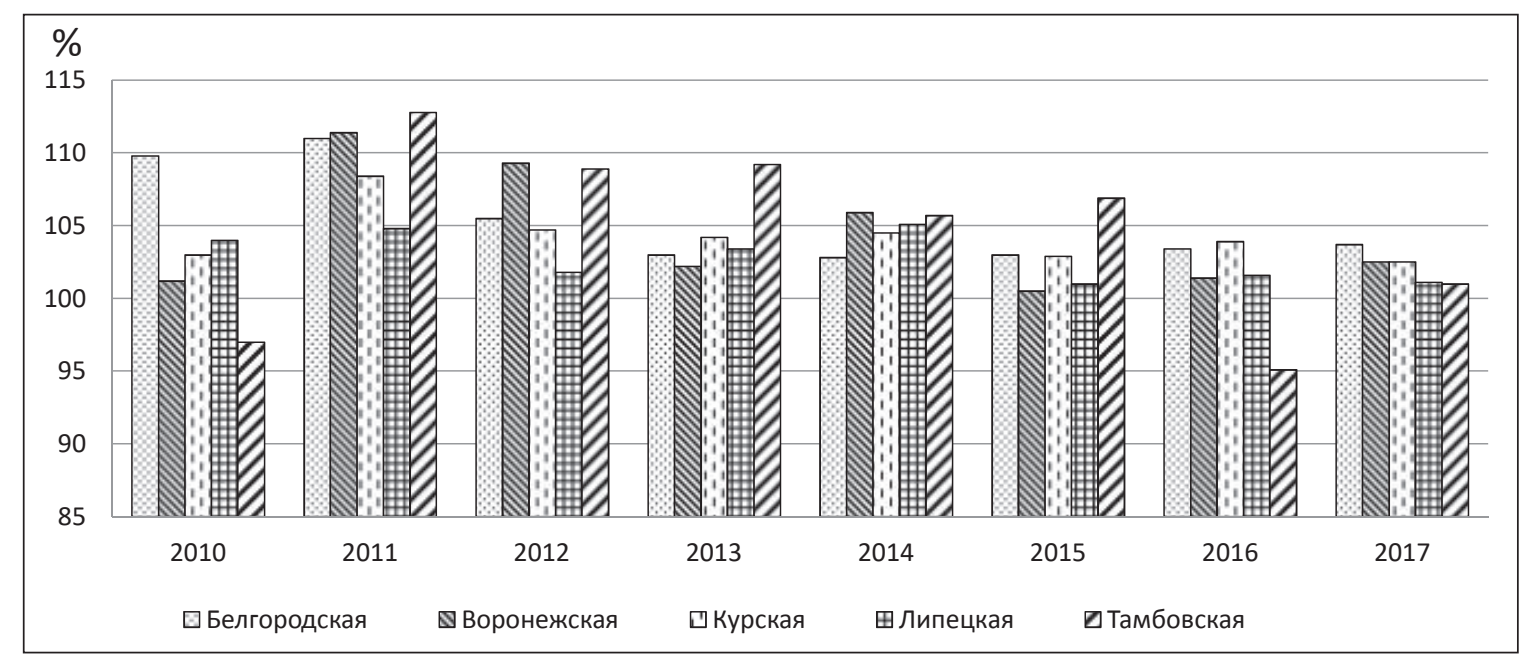

Puc.1. Индекс физического объема ВРП для областей ЦЧР, в \% (построен по [10]) [Fig. 1. GRP physical volume index for the regions of the Central Black Soil District, in \% (built according to [10].)

кой области - это 2013 год, в Курской в 2012-2014 годы и Тамбовской 2014 год. Во всех остальных случаях использование математического моделирования на основе длинных и средних рядов даст заведомо неточный результат. Поэтому неравномерность развития не позволяет в полной мере использовать возможности моделирования на основе тренда. Неравномерность социально-экономического развития при определенном уровне колебаний делает бессмысленным составление такого типа моделей. Надо отметить, моделирование только математическое, без учета факторов социально-экономического развития, не позволяет разрабатывать по настоящему адекватные модели, которые в должной степени реализуют существующие тенденции. На примере ЦЧР видно, как регионы с разным уровнем экономического развития имеют близкие темпы развития. И, наоборот, в регионах с одинаковыми показателями развития уровни динамики будут отличаться. Самое главное, внутренний характер развития при одинаковых темпах может быть разным. Это в значительной степени ухудшает прикладные возможности математических моделей и их практическое применение для прогнозирования. Поэтому необходимо знание факторов социально-экономического развития, которые дают возможность адекватно оценить процессы и тенденции.

Объектом нашего исследования является социально-экономическая система Центрально-Черноземного района. Предметом исследования - социально-экономическое развитие района. Цель статьи - математическое моделирование хозяйства ЦЧР в условиях неопределенности развития. Для этого нами были поставлены и решены следующие основные задачи: рассчитали показатели развития
ЦЧР, построили модели разного типа на основе косвенных приростов по коротким рядам показателям развития хозяйства, оценили их недостатки и преимущества, провели оценку их точности. ЦЧР в настоящее время - прежде всего, агропромышленный район, для которого характерна неравномерность развития из-за погодных условий. При этом многое зависит от специализации хозяйства и от уровня развития производства. Так, в 2010 году вследствие жары и засухи объем сельскохозяйственной продукции во всех областях ЦЧР упал больше, чем на 25 \%. Особенно в Воронежской и Тамбовской областях, кроме Белгородской области, где он остался на уровне 2009 года. Наблюдалось снижение объемов пищевой промышленности, кроме Белгородской области, где она увеличилась.

Динамические ряды объемов промышленной и сельскохозяйственной продукции, объема ВРП ЦЧР не имеют постоянной четкой зависимости (линейной, гиперболической, экспоненциальной), так как наблюдаются значительные колебания внутри этих рядов. Поэтому неоднородность рядов не дает возможности применять регрессионные модели для прогнозирования многомерных процессов. Так, построенная нами многомерная модель для прогноза развития ВРП, промышленности и сельского хозяйства ЦЧР не обладает должной адекватностью [8].

Короткие временные ряды развития крупного региона создают возможности для построения моделей, отражающих динамику развития, но будут недостоверны с точки зрения оценок адекватности статистических моделей. Следовательно, надо найти не новые способы, а новую основу моделирования. По нашему мнению, необходимо использовать для математического моделирова- 
ния те свойства систем, которые можно применить и в неопределенном развитии. В данном случае основными свойствами систем являются взаимосвязанность и устойчивость, поэтому вполне закономерно использовать эти качества, сохраняя пропорции между отраслями в развитии региона.

\section{МАТЕРИАЛЫ И МЕТОДЫ}

Для математического моделирования ЦЧР мы используем такие качества региональной системы как взаимосвязанность элементов и подсистем района.

Моделирование хозяйства ЦЧР в условиях неравномерности и неопределенности социально-экономического развития требует использования особых моделей сверхкороткого ряда. С одной стороны, это показывает новизну работы, но, с другой, - ограничивает применение адаптивного моделирования.

На развитие социально-экономических систем влияют следующие четыре главные неопределенности. Первая. Природная неопределенность, которая главным образом вызывается погодными климатическими условиями, а также чрезвычайными факторами - наводнениями, землетрясениями и т.д. Вторая. Концеептуальная неопределенность, связанная с нечеткими и неправильными представлениями о потенциале и путях развития объекта, что формирует искаженную стратегию управления. Третья. Стратегическая неопределенность зависит от политической ситуации в мире, конкуренции между странами и регионами. На развитии хозяйства также сказывается четвертая поведенческая неопределенность, которая обусловлена непредсказуемым поведением людей.

Информационной базой явились материалы, представленные в официальном издании Федеральной службы государственной статистики (Росстат) - «Регионы России» за 2019 год и материалы территориальных органов федеральной службы государственной статистики Белгородской, Воронежской, Курской, Липецкой и Тамбовской областей. Для выполнения расчетов статистические данные были обобщены, систематизированы и структурированы.

Для достижения поставленной цели применялись сравнительный, структурный, математический и аналитический методы исследования. В настоящее время разработано множество методов экономико-математического моделирования для анализа и прогнозирования состояния социально-экономических образований, в том числе многоуровневых пространственных систем [5, 6, 7]. Из-за пандемии, социально-экономического давления на РФ (ОПЕК, США, ЕС) наблюдается еще большая неопределен- ность будущего развития. В данной ситуации любая модель будет иметь те или иные недостатки, но при этом все же необходимо выбрать те модели, которые будут максимально адекватны существующей реальности. При моделировании хозяйства ЦЧР мы применяли различные виды матричного предикторного моделирования. Сложно спорить, что в хозяйстве региона существует взаимодействие между его территориальными и отраслевыми элементами (предприятиями и отраслями), а структура региональной социально-экономической системы обладает определенной устойчивостью. Поэтому на протяжении некоторого периода времени структура косвенных темпов приростов прогнозируемых показателей может оставаться почти неизменной $[1-4,6]$.

Все модели, созданные с помощью длинных рядов в той или иной степени, связаны с прямым приростом факторных показателей и аргумента. В тех моделях, которые основаны на использовании коротких и даже сверхкоротких рядов (достаточно одного временного периода), на первое место выходит постулат о структурном взаимодействии отраслей хозяйства. Наверное, сложно спорить, что от объема сельскохозяйственной продукции зависит в ЦЧР объем производства промышленной продукции (особенно пищевой) и ВРП, и наоборот. Эта взаимосвязь выражается косвенными темпами приростов, которые представляют собой абсолютный прирост каждой из отраслей к общему объему продукции всех остальных отраслей. Например, прирост сельскохозяйственной продукции к общему объему промышленного производства и объему ВРП, или прирост промышленной продукции к объему сельскохозяйственной продукции и т.д. Поэтому в условиях неопределенности развития, по нашему мнению, наиболее объективным показателем социально-экономической действительности является стабильная взаимосвязь между подсистемами (отраслями). Оценить уровень взаимосвязи между ними можно именно на основе косвенных темпов приростов.

При этом в математическом моделировании коротких рядов мы используем матричное моделирование косвенных приростов, как наиболее подходящие. Матричные модели позволяют в одном контексте рассматривать синхронные и диахронные явления и процессы, формирующиеся в обществе [7, 9].

Особенности построения моделей, обеспечивающих сохранение структуры объекта в условиях неопределенности и неравномерности развития, мы покажем с помощью наиболее простой модели на основе матричного предиктора. 


\section{РЕЗУЛЬТАТЫ И ОБСУЖДЕНИЕ}

Матричный предиктор можно использовать даже тогда, когда нет возможности построения любой статистической модели, например, в случае сверхкоротких рядов, когда имеются показатели лишь двух наблюдений (в момент времени $\mathrm{t}$ и в момент времени t-1). Соответственно получим два показателя $x_{t i}$ и $x_{t-1 i}$, a $\Delta x_{t i}-$ изменение (прирост) $i$-го показателя. Дальше мы объективно предполагаем, что в социально-экономической системе (в данном случае в ЦЧР) изменение любого показателя связано с объемами производства других показателей. При этом примем как постулат, что воздействие показателей достаточно равномерное, среди них нет явно преобладающих. Тогда можно использовать косвенные темпы прироста

$$
v_{i j}=\frac{\Delta x_{t i}}{x_{t j}},
$$

Так как на формирование прироста все показатели оказывают равномерное воздействие, то, разделив $v_{i j}$ на $(n-1)$, мы получим ту долю в приросте $i$-го показателя, которая сформирована под воздействием $j$-го $[1-3,6]$. Затем покажем прирост $\Delta x_{t i}$ как сумму произведений:

$$
\Delta x_{t i}=\frac{1}{n-1} \sum_{j \neq i} v_{i j} x_{t j} .
$$

Так как $\Delta x_{t i}=x_{t i}-x_{t-1 i}$, то:

$$
x_{t i}=x_{t-1 i}+\frac{1}{n-1} \sum_{j \neq i} v_{i j} x_{t j}, \quad i=\overline{1, n} .
$$

Обозначим систему уравнений (3) через матрицы:

$$
\mathbf{x}_{t}=\left(\begin{array}{c}
x_{t 1} \\
x_{t 2} \\
\vdots \\
x_{t n}
\end{array}\right), \quad \mathbf{V}=\frac{1}{n-1}\left(\begin{array}{cccc}
0 & v_{12} & \ldots & v_{1 n} \\
v_{21} & 0 & \ldots & v_{2 n} \\
\vdots & \vdots & \ldots & \vdots \\
v_{n 1} & v_{n 2} & \ldots & 0
\end{array}\right),
$$

или в компактном виде:

$$
\mathbf{x}_{t}=\mathbf{x}_{t-1}+\mathbf{V} \mathbf{x}_{t} .
$$

Выразим неизвестный вектор $\mathrm{x}_{t}$, это ряд показателей неопределенного прогнозного (будущего) года, через показатели базового года:

$$
\mathbf{x}_{t}=(\mathbf{I}-\mathbf{V})^{-1} \mathbf{x}_{t-1},
$$

где I является единичной матрицей.

Обратная матрица (I-V) $)^{-1}$ является матричным мультипликатором, главным элементом модели. Именно он показывает величину изменения объектов из состояния в момент времени (t-1) в состояние момента времени (t). Внедиагональные элементы мультипликатора представляют собой косвенные темпы роста показателей развития хозяйства, а диагонали, соответственно, - прямые темпы роста.

1. Построение матричного предиктора процесса социально-экономического развития ЦЧР. Сначала мы рассчитали для построения детерминированного матричного предиктора абсолютные приросты за 2016 и 2017 гг. по данным таблицы 1.

Таблиия 1

Основные показатели развития хозяйства ЦЧР, млн. руб.

[Table 1. Main indicators of economic development of the CBSR, million roubles]

\begin{tabular}{|c|c|c|c|}
\hline & $\begin{array}{c}\text { Валовой региональный продукт / } \\
\text { Gross regional product }\end{array}$ & $\begin{array}{c}\text { Обрабатывающая промышленность / } \\
\text { Manufacturing industry }\end{array}$ & $\begin{array}{c}\text { Сельское хозяйство / } \\
\text { Agriculture }\end{array}$ \\
\hline 2016 & 2718212 & 1876137 & 764960 \\
\hline 2017 & 2886500 & 1990509 & 764962 \\
\hline 2018 & 3088570 & 2156967 & 825571 \\
\hline
\end{tabular}

Как мы видим из таблицы, показатели развития сельского хозяйства за эти годы почти одинаковы и абсолютного прироста практически нет.

$$
\Delta \mathrm{x}_{2}=\left(\begin{array}{c}
168288 \\
114372 \\
2
\end{array}\right),
$$

В этом случае построить достоверную статистическую модель невозможно. При этом по-

$$
\begin{gathered}
\mathrm{v}_{12}=\frac{\Delta x_{21}}{x_{22}}=0,0845, \mathrm{v}_{13}=\frac{\Delta x_{21}}{x_{23}}=0,2200, \mathrm{v}_{21}=\frac{\Delta x_{22}}{x_{21}}=0,0396, \mathrm{v}_{23}=\frac{\Delta x_{22}}{x_{23}}=0,1495, \\
\mathrm{v}_{31}=\frac{\Delta x_{23}}{x_{21}}=6,9288 \mathrm{E}-07, \mathrm{v}_{32}=\frac{\Delta x_{23}}{x_{22}}=1,0048 \mathrm{E}-06,
\end{gathered}
$$

строение нашей модели затрудняется, так как ряд косвенных приростов будут незначительными, но данные не выбирают. Более того, это показывает большие возможности подобного моделирования. Полученные абсолютные приросты мы используем для расчета косвенных темпов прироста: 
$\mathbf{V}_{2}=\left(\begin{array}{ccc}0 & 0,0423 & 0,1100 \\ 0,0198 & 0 & 0,0748 \\ 3,4644 \mathrm{E}-07 & 0,5024 \mathrm{E}-06 & 0\end{array}\right)$

На основе полученной матрицы вычислим предиктор.

Сначала определим величину матрицу:

$\mathbf{I}-\mathbf{V}_{\mathbf{2}}=\left(\begin{array}{ccc}1 & -0,0423 & -0,1100 \\ -0,0198 & 1 & -0,0748 \\ -3,4644 \mathrm{E}-07 & -0,5024 \mathrm{E}-06 & 1\end{array}\right)$,

Так как главный определитель $\Delta=$

$=\left|\begin{array}{ccc}1 & -0,0423 & -0,1100 \\ -0,0198 & 1 & -0,0748 \\ -3,4644 \mathrm{E}-07 & -0,5024 \mathrm{E}-06 & 1\end{array}\right|=$

$=1-0,0198 * 0,0423=0,9992 \neq 0$, то $\mathbf{I}-\mathbf{V}_{2}$ имеет обратную матрицу. Для нахождения обратной матрицы, вычислим алгебраические дополнения ко всем элементам матрицы $\mathbf{I}-\mathbf{V}_{2}$ :

$$
\begin{aligned}
& \mathrm{A}_{11}=\left|\begin{array}{cc}
1 & -0,0748 \\
-0,5024 \mathrm{E}-06 & 1
\end{array}\right|=1, \\
& \mathrm{~A}_{21}=-\left|\begin{array}{cc}
-0,0423 & -0,1100 \\
-0,5024 \mathrm{E}-06 & 1
\end{array}\right|=0,0423, \\
& \mathrm{~A}_{31}=\left|\begin{array}{cc}
-0,0423 & -0,1100 \\
1 & -0,0748
\end{array}\right|=0,1132, \\
& \mathrm{~A}_{12}=-\left|\begin{array}{cc}
-0,0198 & -0,0748 \\
-3,4644 \mathrm{E}-07 & 1
\end{array}\right|=0,0198, \\
& \mathrm{~A}_{22}=\left|\begin{array}{cc}
1 & -0,1100 \\
-3,4644 \mathrm{E}-07 & 1
\end{array}\right|=1,
\end{aligned}
$$

$$
\begin{aligned}
& \mathrm{A}_{32}=-\left|\begin{array}{cc}
1 & -0,1100 \\
-0,0198 & -0,0748
\end{array}\right|=0,0770, \\
& \mathrm{~A}_{13}=\left|\begin{array}{cc}
-0,0198 & 1 \\
-3,4644 \mathrm{E}-07 & -0,5024 \mathrm{E}-06
\end{array}\right|=0, \\
& \mathrm{~A}_{23}=-\left|\begin{array}{cc}
1 & -0,0423 \\
-3,4644 \mathrm{E}-07 & -0,5024 \mathrm{E}-06
\end{array}\right|=0, \\
& \mathrm{~A}_{33}=\left|\begin{array}{cc}
1 & -0,0423 \\
-0,0198 & 1
\end{array}\right|=0,9992 .
\end{aligned}
$$

Отсюда обратная матрица:

$\mathbf{A}_{2}=(\mathbf{I}-\mathbf{V})^{-1}=1 / 0,9992 *\left(\begin{array}{ccc}1 & 0,0423 & 0,1132 \\ 0,0198 & 1 & 0,0770 \\ 0 & 0 & 0,9992\end{array}\right)$,

с помощью которой можно рассчитать постпрогнозные оценки:

$\widehat{\boldsymbol{x}}_{3}=\mathbf{A}_{2} \mathbf{x}_{2}=1 / 0,9992 *\left(\begin{array}{l}3057292,229 \\ 2106563,774 \\ 764350,0304\end{array}\right)=\left(\begin{array}{c}3059740,021 \\ 2108250,374 \\ 764962\end{array}\right)$,

$\widehat{\boldsymbol{x}}_{\mathbf{4}}=\mathbf{A}_{\mathbf{2}} \mathbf{x}_{\mathbf{3}}=1 / 0,9992 *\left(\begin{array}{c}3273264,341 \\ 2281689,653 \\ 824910,543\end{array}\right)=\left(\begin{array}{c}3275885,049 \\ 2283516,466 \\ 825571\end{array}\right)$.

2. Построение нелинейного варианта матричного предиктора процесса социальноэкономического развития ЦЧР. Теперь составим нелинейную модель, для этого надо взять логарифмы показателей развития хозяйства ЦЧР, по ним затем составим таблицу 2.

Таблица 2

Логарифмы показателей развития хозяйства ЦЧР, ед.

[Table 2. Logarithms of economic development indicators of the CBSR, units]

\begin{tabular}{|c|c|c|c|}
\hline & $\begin{array}{c}\text { Валовой региональный продукт / } \\
\text { Gross regional product }\end{array}$ & $\begin{array}{c}\text { Обрабатывающая промышленность / } \\
\text { Manufacturing industry }\end{array}$ & $\begin{array}{c}\text { Сельское хозяйство / } \\
\text { Agriculture }\end{array}$ \\
\hline 2016 & 14,8155 & 14,4447 & 13,5476 \\
\hline 2017 & 14,8756 & 14,5039 & 13,5476 \\
\hline 2018 & 14,9432 & 14,5842 & 13,6238 \\
\hline
\end{tabular}

Характер построения этой модели отличается только тем, что используются абсолютные и косвенные приросты логарифмов:

абсолютные приросты логарифмов $\Delta_{\ln } \mathrm{x}_{2}=\left(\begin{array}{c}0,0601 \\ 0,0592 \\ 0\end{array}\right)$,

косвенные приросты логарифмов:

$$
\mathbf{V}_{\ln 2}=\left(\begin{array}{ccc}
0 & 0,0021 & 0,0022 \\
0,0020 & 0 & 0,0022 \\
0 & 0 & 0
\end{array}\right) \text {. }
$$

В дальнейшем построение модели совпадает с предыдущим случаем, также рассчитаем предиктор:

$$
\mathbf{I}-\mathbf{V}_{\ln 2}=\left(\begin{array}{ccc}
1 & -0,0021 & -0,0022 \\
-0,0020 & 1 & -0,0022 \\
0 & 0 & 1
\end{array}\right),
$$

алгебраические дополнения к ней равны:

$$
\begin{aligned}
& A_{11}=\left|\begin{array}{cc}
1 & -0,0022 \\
0 & 1
\end{array}\right|=1, \\
& A_{21}=-\left|\begin{array}{cc}
-0,0021 & -0,0022 \\
0 & 1
\end{array}\right|=0,0021,
\end{aligned}
$$




$$
\begin{aligned}
& A_{31}=\left|\begin{array}{cc}
-0,0021 & -0,0022 \\
1 & -0,0022
\end{array}\right|=0,0022, \\
& A_{12}=-\left|\begin{array}{cc}
-0,0020 & -0,0022 \\
0 & 1
\end{array}\right|=0,0020, \\
& A_{22}=\left|\begin{array}{cc}
1 & -0,0022 \\
0 & 1
\end{array}\right|=1, \\
& A_{32}=-\left|\begin{array}{cc}
1 & -0,0022 \\
-0,0020 & -0,0022
\end{array}\right|=0,0022, \\
& A_{13}=\left|\begin{array}{cc}
-0,0020 & 1 \\
0 & 0
\end{array}\right|=0, \\
& A_{23}=-\left|\begin{array}{cc}
1 & -0,0021 \\
0 & 0
\end{array}\right|=0, \\
& A_{33}=\left|\begin{array}{cc}
1 & -0,0021 \\
-0,0020 & 1
\end{array}\right|=1 .
\end{aligned}
$$

Отсюда обратная матрица равна:

$$
\mathbf{A}_{\ln 2}=\left(\mathbf{I}-\mathbf{V}_{\ln 2}\right)^{-\mathbf{1}}=\left(\begin{array}{ccc}
1 & 0,0021 & 0,0022 \\
0,0020 & 1 & 0,0022 \\
0 & 0 & 1
\end{array}\right) .
$$

Затем рассчитаем прогнозные логарифмы:

$$
\begin{aligned}
& \ln \widehat{\boldsymbol{x}}_{\mathbf{3}}=\mathbf{A}_{\ln 2} \ln \mathbf{x}_{2}=\left(\begin{array}{l}
14,9359 \\
14,5635 \\
13,5476
\end{array}\right) \text { и } \\
& \ln \widehat{\boldsymbol{x}}_{\mathbf{4}}=\mathbf{A}_{\ln 2} \ln \mathbf{x}_{3}=\left(\begin{array}{l}
15,0038 \\
14,6441 \\
13,6238
\end{array}\right),
\end{aligned}
$$

а потенцирование полученных логарифмов позволяет получить количественную оценку прогнозных показателей:

$$
\widehat{\boldsymbol{x}}_{\mathbf{3}}=\left(\begin{array}{c}
3066048,019 \\
2112747,941 \\
764976,199
\end{array}\right) \text { и } \widehat{\widehat{\boldsymbol{x}}}_{\mathbf{4}}=\left(\begin{array}{c}
3281463,271 \\
2290086,162 \\
825545,782
\end{array}\right) \text {. }
$$

3. Построение матричного предиктора с регулируемым темпом роста процесса социально-экономического развития ЦЧР. Конечно, неопределенность развития при сохранении структуры объектов подразумевает и непременное изменение абсолютных приростов, когда используется линейная модель, а также и изменение темпов роста - тогда применяются нелинейные модели. Некоторые изменения вполне можно спрогнозировать, зная социально-экономические тенденции и особенности развития. Для этого необходимо с помощью экспертов или анализа динамики еще до построения модели рассчитать коэффициент коррекции. Его же можно получить в результате анализа уже построенной модели, сравнивая по- лученные прогнозные данные с реальными. За счет корректировки темпов роста можно получить более адекватную модель.

Для регулирования (настройки) модели используем параметр $\rho$, на его основе можно скорректировать динамику исследуемых показателей и прогноза. В этом случае:

$$
x_{i t}=x_{i t-1} \cdot\left(\frac{x_{i t}}{x_{i t-1}}\right)^{\rho}, \quad i=\overline{1, m}
$$

или в логарифмах:

$$
\ln x_{i t}=\ln x_{i t-1}+\rho\left(\ln x_{i t}-\ln x_{i t-1}\right), \quad i=\overline{1, m} .
$$

Корректирующий параметр $\rho$ всегда больше 0 . Если наблюдается снижения темпов роста, то он будет меньше 1,0, в случае увеличения темпов роста параметр должен быть больше 1,0.

Для более яркого сравнения с другими матричными предикторными моделями используем параметр $\rho$, так как в условиях кризиса обычно наблюдается падение производства. Можно было взять другой показатель, который более точно соответствует реальности, но в данном случае такой показатель более четко покажет особенности этой модели. Обычно этот параметр, устанавливается на основе экспертных опросов, «мозгового штурма» и т.д.

Проведем прогнозные расчеты на основе таблицы 1.

Рассчитаем приросты логарифмов показателей таблицы, умноженных на параметр $\rho$ :

$$
\rho \cdot \Delta_{\ln } \mathrm{x}_{2}=\left(\begin{array}{c}
0,0481 \\
0,0474 \\
0
\end{array}\right),
$$

дальше сформируем матрицу косвенных темпов прироста скорректированных логарифмов:

$$
\mathbf{V}_{\ln 2}^{\rho}=\left(\begin{array}{ccc}
0 & 0,0017 & 0,0018 \\
0,0016 & 0 & 0,0018 \\
0 & 0 & 0
\end{array}\right)
$$

Дальше также вычисляем предиктор на основе матрицы:

$$
\begin{aligned}
& \mathbf{I}-\mathbf{V}_{\ln 2}^{\rho}=\left(\begin{array}{ccc}
1 & -0,0017 & -0,0018 \\
-0,0016 & 1 & -0,0018 \\
0 & 0 & 1
\end{array}\right), \\
& A_{11}=\left|\begin{array}{ccc}
1 & -0,0018 \\
0 & 1
\end{array}\right|=1, \\
& A_{21}=-\left|\begin{array}{cc}
-0,0017 & -0,0018 \\
0 & 1
\end{array}\right|=0,0017 \\
& A_{31}=\left|\begin{array}{cc}
-0,0017 & -0,0018 \\
1 & -0,0018
\end{array}\right|=0,0018
\end{aligned}
$$


$A_{12}=-\left|\begin{array}{cc}-0,0016 & -0,0018 \\ 0 & 1\end{array}\right|=0,0016$,

$\mathrm{A}_{22}=\left|\begin{array}{cc}1 & -0,0018 \\ 0 & 1\end{array}\right|=1$,

$A_{32}=-\left|\begin{array}{cc}1 & -0,0018 \\ -0,0016 & -0,0018\end{array}\right|=0,0018$,

$A_{13}=\left|\begin{array}{cc}-0,0016 & 1 \\ 0 & 0\end{array}\right|=0$,

$A_{23}=-\left|\begin{array}{cc}1 & -0,0017 \\ 0 & 0\end{array}\right|=0$,

$\mathrm{A}_{33}=\left|\begin{array}{cc}1 & -0,0017 \\ -0,0016 & 1\end{array}\right|=1$.

Подставляя значения алгебраических дополнений, получим обратную матрицу:
$\mathbf{A}_{\ln 2}^{\rho}=\left(\mathbf{I}-\mathbf{V}_{\ln 2}^{\rho}\right)^{-1}=\left(\begin{array}{ccc}1 & 0,0017 & 0,0018 \\ 0,0016 & 1 & 0,0018 \\ 0 & 0 & 1\end{array}\right)$

На ее основе рассчитаем логарифмы:

$\ln ^{\rho} \widehat{\boldsymbol{x}}_{\mathbf{3}}=\mathbf{A}_{\ln 2}^{\rho} \ln \mathbf{x}_{2}=\left(\begin{array}{c}14,9247 \\ 14,5521 \\ 13,5476\end{array}\right)$ и

$\ln ^{\rho} \widehat{\boldsymbol{x}}_{\mathbf{4}}=\mathbf{A}_{\ln 2}^{\rho} \ln \mathbf{x}_{3}=\left(\begin{array}{l}14,9925 \\ 14,6326 \\ 13,6238\end{array}\right)$,

потенцируя логарифмы, получим результат:

$$
\widehat{\boldsymbol{x}}_{\mathbf{3}}=\left(\begin{array}{c}
3031899,868 \\
2088799,381 \\
764976,199
\end{array}\right) \text { и } \widehat{\boldsymbol{x}}_{\mathbf{4}}=\left(\begin{array}{c}
3244591,454 \\
2263901,024 \\
825545,782
\end{array}\right) \text {. }
$$

Полученные результаты прогнозных моделей показаны в таблице 3.

Таблийа 3

Результаты матричного предикторного моделирования

[Table 3. Results of matrix predictor modelling]

\begin{tabular}{|c|c|c|c|}
\hline \multirow[b]{2}{*}{ Показатели / Indicators } & \multicolumn{3}{|c|}{$\begin{array}{c}\text { Показатели развития ЦЧР в } 2018 \text { г. / } \\
\text { Indicators of the development of the CBSR in } 2018\end{array}$} \\
\hline & BPП / GRP & $\begin{array}{c}\text { Обрабатывающая промыш- } \\
\text { ленность / Manufacturing } \\
\text { industry } \\
\end{array}$ & $\begin{array}{c}\text { Сельское хозяйство / } \\
\text { Agriculture }\end{array}$ \\
\hline $\begin{array}{l}\text { Фактическое значение, млн. руб. / } \\
\text { Actual value, million roubles }\end{array}$ & 3088570 & 2156967 & 825571 \\
\hline \multicolumn{4}{|c|}{$\begin{array}{c}\text { Детерминированный матричный предиктор (ДМП) / } \\
\text { Deterministic matrix predictor (DMP) }\end{array}$} \\
\hline $\begin{array}{l}\text { Постпрогнозная оценка, млн. руб. / } \\
\text { Post-forecast estimate, million roubles }\end{array}$ & 3059740 & 2108250 & 764962 \\
\hline Ошибка, \% / Mistake, \% & $-0,9$ & $-2,3$ & $-7,3$ \\
\hline \multicolumn{4}{|c|}{$\begin{array}{c}\text { Нелинейный вариант детерминированного матричного предиктора (НВДМП) / } \\
\text { A non-linear version of a deterministic matrix predictor (NVDMP) }\end{array}$} \\
\hline $\begin{array}{l}\text { Постпрогнозная оценка, млн. руб. / } \\
\text { Post-forecast estimate, million roubles }\end{array}$ & 3066048 & 2112748 & 764976 \\
\hline Ошибка, \% / Mistake, \% & $-0,73$ & $-2,0$ & $-7,3$ \\
\hline \multicolumn{4}{|c|}{$\begin{array}{c}\text { Матричный предиктор с регулируемым темпом роста (MПсPTP) / } \\
\text { Matrix predictor with adjustable growth rate (MPAGR) }\end{array}$} \\
\hline $\begin{array}{l}\text { Постпрогнозная оценка, млн. руб. / } \\
\text { Post-forecast estimate, million roubles }\end{array}$ & 3031900 & 2088799 & 764976 \\
\hline Ошибка, \% / Mistake, \% & $-1,8$ & $-3,2$ & $-7,3$ \\
\hline $\begin{array}{l}\text { Постпрогнозная оценка, млн. руб. } \\
\text { за } 2019 \text { год (ДМП) / } \\
\text { Post-forecast estimate, million roubles } \\
\text { for } 2019 \text { ((DMP)) }\end{array}$ & 3275885 & 2283516 & 825571 \\
\hline $\begin{array}{l}\text { Постпрогнозная оценка, млн. руб. } \\
\text { за } 2019 \text { год (НВДМП) / } \\
\text { Post-forecast estimate, million roubles } \\
\text { for } 2019 \text { (NVDMP) }\end{array}$ & 3281463 & 2290086 & 825546 \\
\hline
\end{tabular}




\begin{tabular}{|l|l|l|l|}
\hline $\begin{array}{l}\text { Постпрогнозная оценка, млн. руб. } \\
\text { 3a 2019 год (МПсРТP) / } \\
\text { Post-forecast estimate, million roubles } \\
\text { for 2019 (MPAGR) }\end{array}$ & 3244591 & 2263901 & 825546 \\
\hline
\end{tabular}

\section{ВЫВОДЫ}

Сделан анализ проведенного прогнозного моделирования. Результаты моделирования развития промышленности и ВРП имеют достаточно высокую точность, несмотря на почти нулевой абсолютный прирост в объеме сельскохозяйственной продукции. Даже отсутствие прироста в одном из показателей за счет корректировки темпов роста и объективном коэффициенте коррекции не мешает получить адекватную модель, что расширяет возможности применения прогнозных моделей.

На основе нашего исследования можно сделать вывод, что модели на основе кратковременных рядов отражают особенности социально-экономического развития даже в условиях значительной или полной неопределенности динамических процессов и вполне могут использоваться для прогнозного моделирования развития больших территорий (регионов и крупных городских округов). При этом особое значение имеет использование их в сочетании с качественными методами прогнозирования. Наиболее перспективными в условиях неопределенности развития могут стать адаптивные матричные предикторные модели с регулируемыми темпами роста. Для этих моделей необходим чуть более длинный ряд показателей, на основе которых можно настроить корректирующий параметр, так как прогнозную ошибку определяют по фактическим данным. Чтобы получить многомерный адаптивный предиктор как комбинацию текущего и скорректированного предикторов необходимо применение скорректированного предиктора (возможно и не один раз), после вводится настраиваемый параметр. Матричное предикторное моделирование является основой других более сложных и комплексных методов моделирования, в том числе имитационного.

Так как имитационное моделирование основано на методе подражания поведению изучаемых объектов, в условиях неопределенности и неравномерности развития это делает его иногда единственным возможным для применения инструментом моделирования региональных социально-экономических процессов. Точность имитационных моделей зависит от уровня подражания этим процессам. Математические имитационные модели, которые раскрывают характер законов, обеспечи- вающих функционирование экономических объектов, относятся к имитационным системам первого ранга. Модели, которые обеспечивают подражание также в распределении случайных величин, относят ко второму рангу. Современное программное обеспечение позволяет создавать даже имитационные модели третьего ранга, где осуществляется почти полное подражание социально-экономическим процессам развития региона. Развитие математического моделирования делает возможным использовать матричное моделирования как часть комплексных регрессионно-матричных моделей сложных многомерных социально-экономических региональных процессов.

\section{СПИСОК ЛИТЕРАТУРЫ}

1. Давнис В.В. Адаптивное прогнозирование: модели и методы. Воронеж: ВГУ, 1997. 196 с.

2. Давнис В.В., Тинякова В.И. Современные тенденции развития прогностических методов управления экономикой // Материаль международной научно-практической конферениии «Экономическое прогнозирование: модели и методыl», 2005, с. 29-36.

3. Давнис В. В., Зеленцова С.В. Применение матричного предиктора в задачах имитационного моделирования показателей экономического развития региона // Maтериаль Международной научно-практической конференции «Теоретические основы и опыт стратегического планирования развития территорий», 2005, с. 193-197.

4. Давнис В. В., Коротких В. В., Юрова Я. А. Регрессионно-матричная модель многомерных экономических процессов // Современная экономика: проблемы и ремения, 2016, 11 (83), с. 19-29.

5. Зеленцова С. Ю., Кирьянчук В.Е., Крупко А.Э. Многомерное адаптивно-имитационное моделирование в системе прогнозирования социально-экономического развития региона // Вестник Воронежского государственного университета. Серия: Экономика и управление, 2006, № 1, с. 148-154.

6. Крупко А.Э. Некоторые теоретические аспекты географического исследования устойчивого (сбалансированного) развития региона // Вестник Воронежского государственного университета. Серия: География. Геоэкология, 2011, № 2, с. 46-51.

7. Крупко А.Э., Фетисов Ю.М., Нестеров Ю.А., Черкашин А.К. Моделирование сбалансированного социально-экономического развития общественных социально-экономических систем (на примере ЦЧР) // Вестник Воронежского государственного университета. Серия География. Геоэкология, 2016, № 1, с. 5-15.

8. Крупко А.Э., Фетисов Ю. М., Рогозина Р.Е. Проблемы прогнозирования социально-экономического

Вестник ВГУ, Серия: География. Геоэкология, 2021, № 4, 50-59 
развития Центрально-Черноземного района в условиях политической и социально-экономической нестабильности // Бизнес. Образование. Право, 2020, № 1 (50), c. 302-309. DOI: 10256.83/VOLBI.2020.50.181.

9. Черкашин А.К., Истомина Е.А., Владимиров И.Н., Мядзелец А. В., Мясникова С.И., Солодянкина С. В., Трофимова И.Е., Фролов А.А. Гомология и гомотопия географических систем. Новосибирск: Гео, 2009. 351 с.
10. Регионы России. Социально-экономические показатели. 2019: Стат. сб. / Росстат. М., 2019. 1204 с.

Конфликт интересов: Авторы декларируют отсутствие явных и потенциальных конфликтов интересов, связанных с публикацией настоящей статьи.

Поступила в редакциию 12.11.2020

Принята к публикации 23.11.2021

\title{
Mathematical Modelling of the Economy of the Central Black Soil Region
}

\author{
Yu. M. Fetisov, A. E. Krupko ${ }^{凶}$ \\ Voronezh State University, Russian Federation \\ (1, Universitetskaya Sq., Voronezh, 394018)
}

\begin{abstract}
The purpose is to identify the specifics of modelling the economy of the Central Black Soil Region (CBSR) under conditions of uneven and uncertain socio-economic development.

Materials and methods. Structural-systemic, mathematical and analytical research methods were applied in the article. We used various types of matrix predictor models to model the economy of the Central Black Soil Region. The article uses statistical information provided in the official publications of the Federal State Statistics Service - "Regions of Russia" for 2019. In order to perform the calculations, the statistical data have been summarized, systematized and structured.

Results and discussion. Indicators of the development of economies of the region were calculated, models of different types were built by means of indirect increments over short series of indicators of the development of an economy on the basis of a deterministic matrix predictor, their disadvantages and advantages were noted, and the accuracy of the models was evaluated.

Conclusion. Mathematical modelling of the region's economy under conditions of uneven and uncertain socio-economic development on the basis of short and ultra-short series is one of the main directions of modelling complex multidimensional socio-economic regional processes as a separate method and as part of the comprehensive mathematical modelling of socio-economic development of the region.
\end{abstract}

Key words: Central Black Soil region, development, unevenness, uncertainty, deterministic matrix predictor, absolute growth, indirect growth rate.

For citation: Fetisov Yu. M., Krupko A.E. Mathematical Modelling of the Economy of the Central Black Soil Region. Vestnik Voronezskogo gosudarstvennogo universiteta. Seria: Geografia. Geoekologia, 2021, no. 4, pp. 50-59. (In Russ.) DOI: https://doi.org/10.17308/geo.2021.4/3750

\section{REFERENCES}

1. Davnis V.V. Adaptivnoe prognozirovanie: modeli $i$ metody [Adaptive forecasting: models and methods]. Voronezh: VGU, 1997. 196 p. (In Russ.)

2. Davnis V.V., Tinyakova V.I. Sovremennye tendentsii razvitiya prognosticheskikh metodov upravleniya ekonomikoy [Current trends in the development of predictive meth- ods of economic management]. Materialy mezhdunarodnoy nauchno-prakticheskoy konferentsii «Ekonomicheskoe prognozirovanie: modeli i metody», 2005, pp. 29-36. (In Russ.)

3. Davnis V.V., Zelentsova S.V. Primenenie matrichnogo prediktora $\mathrm{v}$ zadachakh imitatsionnogo modelirovaniya pokazateley ekonomicheskogo razvitiya regiona [Application of the matrix predictor in the tasks

(C) Fetisov Yu. M., Krupko A.E., 2021

$\triangle$ Anatoliy E. Krupko, glomer-a@mail.ru

The content is available under Creative Commons Attribution 4.0 License. 
of simulation modeling of indicators of economic development of the region]. Materialy Mezhdunarodnoy nauchno-prakticheskoy konferentsii "Teoreticheskie osnovy $i$ opyt strategicheskogo planirovaniya razvitiya territoriy», 2005. pp. 193-197. (In Russ.)

4. Davnis V.V., Korotkikh V.V., Yurova Ya.A. Regressionno-matrichnaya model' mnogomernykh ekonomicheskikh protsessov [Regression matrix model of multidimensional economic processes]. Sovremennaya ekonomika: problemy i resheniya, 2016, 11 (83), pp. 19-29. (In Russ.)

5. Zelentsova S. Yu., Kir'yanchuk V.E., Krupko A.E. Mnogomernoe adaptivno-imitatsionnoe modelirovanie $\mathrm{V}$ sisteme prognozirovaniya sotsial'no-ekonomicheskogo razvitiya regiona [Multidimensional adaptive simulation modeling in the forecasting system of socio-economic development of the region]. Vestnik Voronezhskogo gosudarstvennogo universiteta. Seria: Ekonomika i upravlenie, 2006, no. 1, pp. 148-154. (In Russ.)

6. Krupko A.E. Nekotorye teoreticheskie aspekty geograficheskogo issledovaniya ustoychivogo (sbalansirovannogo) razvitiya regiona [Some theoretical aspects of geographical research of sustainable (balanced) development of the region]. Vestnik Voronezhskogo gosudarstvennogo universiteta. Seria: Geografia. Geoekologia, 2011, no. 2, pp. 46-51. (In Russ.)

7. Krupko A.E., Fetisov Yu.M., Nesterov Yu.A., Cherkashin A.K. Modelirovanie sbalansirovannogo sotsial'no-ekonomicheskogo razvitiya obshchestvennykh sotsial'no-ekonomicheskikh sistem (na primere TsChR) [Mod-

\section{Фетисов Юрий Михайлович}

Кандидат физико-математических наук, доцент кафедры рекреационной географии, страноведения и туризма факультета географии, геоэкологии и туризма Воронежского государственного университета, г. Воронеж, Российская Федерация, e-mail: deanery@geogr.vsu.ru

Крупко Анатолий Эммануилович

Кандидат географических наук, доцент кафедры социально-экономической географии и регионоведения факультета географии, геоэкологии и туризма Воронежского государственного университета, г. Воронеж, Российская Федерация, ORCID: 0000-0003-3141-5914, e-mail: glomer-a@mail.ru eling of balanced socio-economic development of public socio-economic systems (using the example of the Central Bank)]. Vestnik Voronezhskogo gosudarstvennogo universiteta. Seria Geografia. Geoekologia, 2016, no. 1, pp. 5-15. (In Russ.)

8. Krupko A. E., Fetisov Yu. M., Rogozina R. E. Problemy prognozirovaniya sotsial'no-ekonomicheskogo razvitiya Tsentral'no-Chernozemnogo rayona $\mathrm{v}$ usloviyakh politicheskoy i sotsial'no-ekonomicheskoy nestabil'nosti [Problems of forecasting the socio-economic development of the Central Chernozem region in conditions of political and socio-economic instability]. Biznes. Obrazovanie. Pravo, 2020, no. 1 (50), pp. 302-309. (In Russ.) DOI: 10256.83/VOLBI.2020.50.181.

9. Cherkashin A. K., Istomina E.A., Vladimirov I. N., Myadzelets A. V., Myasnikova S. I., Solodyankina S. V., Trofimova I.E., Frolov A.A. Gomologiya i gomotopiya geograficheskikh system [Homology and homotopy of geographical systems]. Novosibirsk: Geo, 2009. 351 p. (In Russ.)

10. Regiony Rossii. Sotsial'no-ekonomicheskie pokazateli. 2019: Stat. sb. [Socio-economic indicators. 2019: Stat. sat.]. Rosstat. M., 2019. 1204 p. (In Russ.)

Conflict of interests: The authors declare no information of obvious and potential conflicts of interest related to the publication of this article.

Received: 12.11.2020

Accepted: 23.11.2021

\section{Yuri M. Fetisov}

Cand. Sci. (Phys.-Math.), Associate Professor of the Department of Recreational Geography, Country Studies and Tourism, Faculty of Geography, Geoecology and Tourism, Voronezh State University, Voronezh, Russian Federation, e-mail: deanery@geogr.vsu.ru

Anatoliy E. Krupko

Cand. Sci. (Geogr.), Associate Professor of the Department of Social and Economic Geography and Regional Studies, Faculty of Geography, Geoecology and Tourism, Voronezh State University, Voronezh, Russian Federation, ORCID: https:// orcid.org/0000-0003-3141-5914, e-mail: glomer-a@mail.ru 